\title{
Undergraduate Students' Acceptance of a Reciprocating One-File System for Endodontic Treatment
}

\author{
Benjamin Mahmoodi ${ }^{1}$ Adriano Azaripour ${ }^{1} \quad$ Kawe Sagheb $^{2} \quad$ Keyvan Sagheb ${ }^{3} \quad$ Brita Willershausen ${ }^{1}$ \\ Jens Weusmann ${ }^{1}$
}

${ }^{1}$ Department of Periodontology and Operative Dentistry, Universitätsmedizin Mainz, Johannes-Gutenberg University, Mainz, Germany

2Department of Prosthodontics, Universitätsmedizin Mainz, Johannes-Gutenberg University, Mainz, Germany

${ }^{3}$ Department of Dentomaxillofacial Surgery, Universitätsmedizin Mainz, Johannes-Gutenberg University, Mainz, Germany

\begin{abstract}
Address for correspondence Jens Weusmann, DDS, MSc, Department of Periodontology and Operative Dentistry, Universitätsmedizin Mainz, Johannes Gutenberg University, Augustusplatz 2, 55131 Mainz, Germany (e-mail: jens.weusmann@unimedizin-mainz.de).
\end{abstract}

Eur J Dent:2020;14:393-396

\begin{abstract}
Objectives Reciprocating endodontic one-file systems are a comparatively new method for root canal shaping. Even though the mechanical properties are comparable to modern rotating mechanical systems, data about subjective assessment and application quality are scarce. This study evaluates the reciprocating one-file system in undergraduate education.

Materials and Methods A total of 42 undergraduate students without experience regarding reciprocating file systems filled in a questionnaire in four different points in time $\left(t_{1}-t_{4}\right)$ anonymously. The questionnaire was based on a numerical rating scale ranging from 0 to 10 .

Statistical Analysis A least significant difference post-hoc analysis comparing the group average values was performed. The adjusted level of significance was $p<0.004$ after Bonferroni correction.

Results All rating scores increased after the first theoretical instruction. After the first practical training in artificial root canals in resin blocks and extracted teeth $\left(t_{2}\right)$, the estimation of "time efficiency" ( $p=0.002)$, "handling" $(p<0.001)$, and "overall

\section{Keywords}

- reciprocation

- undergraduate teaching

- endodontics

- one-file system impression" ( $p<0.001)$ improved significantly. The "overall impression" remained constant and showed no significant changes after the first practical training.

Conclusions Reciprocating systems seem to show a good acceptance among first time users. Initial concerns about "work safety" decrease during every step of the educational process. Reciprocating one-file systems are a safe and well-accepted method in undergraduate teaching.
\end{abstract}

\section{Objectives}

The market launch of reciprocating nickel-titanium instruments led to a substantial facilitation of the mechanical root canal preparation. In these systems, usually only one file is necessary for complete preparation, leading not only to a lower expenditure but also to a saving of time, since it made preflaring not mandatory anymore. ${ }^{1-3}$ Complications such as file fracture, canal straightening, or perforation have become rarer and originally, canal anatomy is easier to respect, even in strongly curved canals. ${ }^{4-6}$ Additionally, the critical hygienic treatment of the files can be avoided, since the reciprocating file systems are single-use instruments. 
Regarding the mechanical superiority of rotating nickel-titanium instruments compared with stainless steel hand files, ${ }^{7}$ reciprocating instruments are similarly reliable as rotating instruments. ${ }^{4,8-10}$ Hitherto, the knowledge about subjective assessment and application quality is scarce. ${ }^{11}$

Reciproc Blue (VDW; Munich, Germany) is the latest reciprocating file system and succeeds the Reciproc system (VDW) with improved manufacturing methods. The main differences are the metallurgic properties: a new file generation that includes Reciproc Blue is treated in a complex thermical process that results in a visible layer of titanium oxide on the instrument's surface. This treatment entails improved flexibility and fatigue resistance. ${ }^{12}$ The files have an s-shaped profile with two cutting edges and a noncutting tip. In vitro trials showed superior mechanical characteristics compared with other reciprocating files on the market. ${ }^{13,14}$

Other studies showed the canal preparation with reciprocating systems to be less technique sensitive ${ }^{15}$ and to be less influenced by the practitioner's experience looking at the successful root canal shaping. Even beginners were able to achieve results of reproducible quality with reciprocating systems ${ }^{16}$ and preferred them over rotating instruments. ${ }^{17}$

The aim of our study was to evaluate the Reciproc Blue system in undergraduate education. Within the scope of the practical student course, the Reciproc Blue system was implemented in both theory and practice as well as clinical use. On this occasion, students' confidence in mechanical and physical properties as well as the subjective operational safety was assessed on a basis of a questionnaire in different points in time.

\section{Materials and Methods}

By means of a questionnaire, we recorded the individual assessment of 42 undergraduate dentistry students. Hitherto, none of the participants had any experience regarding reciprocating endodontic files. The ethics committee allowed the proceeding (Ref. No. 837.488.17).

A new protocol for canal preparation was introduced for the students in their final year in the scope of the clinical students' course for operative dentistry. Here, the Reciproc Blue system was to be used as the standard instrument for the endodontic first-line therapy following theoretical and practical training. The preparation protocol was carried out according to manufacturer specifications.

The evaluation was performed using a numerical rating scale ranging from 0 to $10(0=$ no confidence; $10=$ highest confidence in the respective attribute). The questionnaire contained 10 questions regarding the three main attributes: "handling," "work safety," and "time efficiency," as well as the "overall impression" of the system.

The questionnaire was filled in anonymously at four different times $\left(t_{1}-t_{4}\right)$ during the course. The first inquiry was after the theoretical explanation of the system $\left(t_{1}\right)$, and the second after the first use in resin blocks with artificial root canals $\left(t_{2}\right)$. The further inquiries were after the first clinical use $\left(t_{3}\right)$ and at the end of the term after repeated clinical application $\left(t_{4}\right)$.

The data were recorded using Microsoft Excel 2016 (Microsoft, Redmond, WA, USA) and SPSS software version 22; IBM Corp, Armonk, NY, USA) and statistically evaluated. By means of a least significant difference post hoc analysis, the group average values were compared. The adjusted level of significance was $p<0.004$ after Bonferroni correction.

\section{Results}

Altogether, 42 undergraduate students of dentistry without any experience with reciprocating file systems took part in this prospective study. At no point of time, a file fracture was observed. All primary endodontic treatments passed without complications. Every student treated at least six patients' root canals during the course.

In all rating categories, an increase of the score became apparent after the first theoretical instruction $\left(t_{1}\right)$ (-Table 1). After the first practical training in artificial root canals in resin blocks and extracted teeth $\left(t_{2}\right)$, the estimation of "time efficiency" ( $p=0.002)$, "handling" $(p<0.001)$, and "overall impression" ( $p<0.001$ ) improved significantly (-Fig. 1) (-Table 1). The "overall impression" remained constant and showed no significant changes after the first practical training.

Regarding "work safety" of the file, a significant growth of the trust levels was observed after the first practical training $\left(t_{2}\right)$. At the end of the term $\left(t_{4}\right)$, the confidence had again increased compared with the first clinical use $\left(t_{3}\right)(p=0.001)$ ( - Table 1 ).

\section{Discussion}

In every category, a significant increase of confidence after first practical model training was noticeable. This level remained steady regarding "handling," "time effectiveness," and "overall impression." For "work safety," a further significant increase was recorded at the end of the term after several times of clinical use. The data indicate the initial reluctance toward the reciprocating system, in particular, toward "work safety." This can be explained by the fact that

Table 1 Mean values and standard deviations of the estimations in the respective points in time

\begin{tabular}{|l|l|l|l|l|}
\hline & $t_{1}$ & $t_{2}$ & $t_{3}$ & $t_{4}$ \\
\hline Handling & $7.1 \pm 2.07$ & $8.27 \pm 1.5$ & $8.02 \pm 1.32$ & $8.08 \pm 1.17$ \\
\hline Work safety & $5.78 \pm 2.03$ & $7.02 \pm 1.77$ & $7.17 \pm 1.53$ & $7.61 \pm 1.19$ \\
\hline Time efficiency & $7.71 \pm 1.49$ & $8.63 \pm 1.38$ & $8.43 \pm 1.11$ & $8.4 \pm 1.32$ \\
\hline Overall impression & $6.05 \pm 1.78$ & $7.36 \pm 1.68$ & $7.43 \pm 1.4$ & $7.86 \pm 1.39$ \\
\hline
\end{tabular}




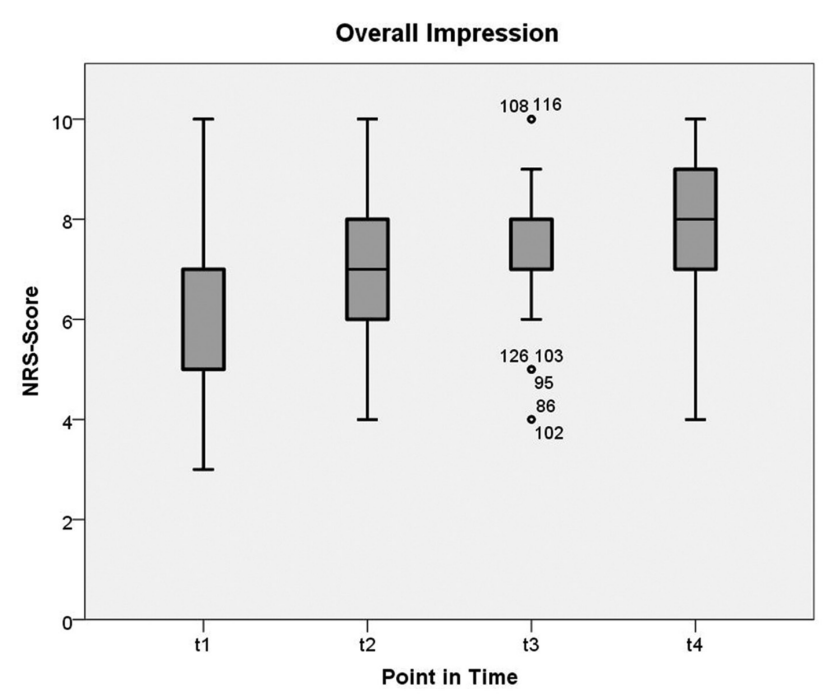

Fig. 1 Overall impression of the tested reciprocating one-file system in different points in time. NRS, numerical rating scale.

the participants had no experience with reciprocating file systems so far. Heretofore, mechanical endodontic preparation had been taught by means of a rotating file system.

The use of rotating nickel-titanium instruments in clinical education is widely established, and it was able to show good results in comparison to manual preparation.

Data dealing with reciprocation in clinical education do not exist until the present day, although Muñoz et al showed that a sufficient resin block canal preparation is possible for unexperienced users. ${ }^{16}$ In an investigation by Kwak et al, 81 undergraduate students without any experience prepared each two molars with stainless steel hand files, a rotating nickel-titanium file system and a reciprocating nickel-titanium file system. Here, the students clearly preferred two mechanical nickel-titanium systems over manual preparation, whereby twice as many $(n=55)$ favored the reciprocating file system over the rotating system. ${ }^{17}$

Our study collective was already familiar with a rotating mechanical file system and started with a comparatively skeptical attitude toward the new system that shriveled during the term. A study by Bartols et al reported differing results; in this trial, 64 unexperienced students were offered tow rotating (FlexMaster and Mtwo) and one reciprocating (Reciproc) file system were offered for the preparation of collectively 186 model root canals. As a result, the most centric preparation was seen in the reciprocating group, even though the students assessed a rotating file system (Mtwo) as the easiest one to use. ${ }^{11}$

All comparing studies have in common that mechanical systems are widely preferred over manual preparation, which is consistent with our observations. The reasons for the good acceptance might be flexibility and fracture resistance of the Reciproc blue system. ${ }^{3}$ Furthermore, the fact that the tested system allows a preparation without glide path ${ }^{1,2}$ might have eased the use especially for the less trained clinician, even though in the students' education, a glide path was always created interalia due to the increased fracture strength. ${ }^{18}$ As a future recommendation, a comparison between rotating and reciprocating systems among students would be surely of interest. Despite the limitations of a survey assessment study, our results show that Reciproc blue is a safe and well-accepted system even for less-experienced users. It is to be expected that the addition of mechanical systems will replace the traditional manual preparation in teaching more and more.

\section{Conflict of Interest}

The authors declare no conflict of interest related to this study. B.M., A.A., B.W., and J.W. report nonfinancial support from VDW, Munich, Germany, during the conduct of the study for the Department of Periodontology and Operative Dentistry.

\section{Acknowledgment}

The authors would like to thank VDW, Munich, Germany for providing the endodontic files and resin blocks.

\section{References}

1 Rodrigues E, De-Deus G, Souza E, Silva EJ. Safe mechanical preparation with reciprocation movement without glide path creation: result from a pool of 673 root canals. Braz Dent J 2016;27(1):22-27

2 Bartols A, Robra BP, Walther W. The ability of Reciproc instruments to reach full working length without glide path preparation: a clinical retrospective study. PeerJ 2017;5:e3583

3 De-Deus G, Arruda TE, Souza EM, et al. The ability of the Reciproc R25 instrument to reach the full root canal working length without a glide path. Int Endod J 2013;46(10):993-998

4 Bürklein S, Hinschitza K, Dammaschke T, Schäfer E. Shaping ability and cleaning effectiveness of two single-file systems in severely curved root canals of extracted teeth: Reciproc and WaveOne versus Mtwo and ProTaper. Int Endod 2012;45(5):449-461

5 da Frota MF, Espir CG, Berbert FL, et al. Comparison of cyclic fatigue and torsional resistance in reciprocating single-file systems and continuous rotary instrumentation systems. J Oral Sci 2014;56(4):269-275

6 Saber SE, Nagy MM, Schäfer E. Comparative evaluation of the shaping ability of WaveOne, Reciproc and OneShape single-file systems in severely curved root canals of extracted teeth. Int Endod J 2015;48(1):109-114

7 Celik D, Taşdemir T, Er K. Comparative study of 6 rotary nickel-titanium systems and hand instrumentation for root canal preparation in severely curved root canals of extracted teeth. J Endod 2013;39(2):278-282

8 Machado R, Comparin D, Back ED, Garcia LDFR, Alberton LR. Residual smear layer after root canal instrumentation by using Niti, M-Wire and CM-Wire instruments: a scanning electron microscopy analysis. Eur J Dent 2018;12(3):403-409

9 Kesim B, Sagsen B, Aslan T. Evaluation of dentinal defects during root canal preparation using thermomechanically processed nickel-titanium files. Eur J Dent 2017;11(2):157-161

10 Arruda EDS, Sponchiado-Júnior EC, Pandolfo MT, Fredson MAC, Roberi Garcia LDF, Marques AA. Apical transportation and centering ability after root canal filling removal using reciprocating and continuous rotary systems: a CBCT study. Eur J Dent 2019;13(4):613-618

11 Bartols A, Christofzik DW, Krummel M, et al. Assessment of different root canal preparation techniques with rotary nickel-titanium instruments by novice students. Dent J (Basel) 2018;6(3):E46 
12 De-Deus G, Silva EJ, Vieira VT, et al. Blue thermomechanical treatment optimizes fatigue resistance and flexibility of the Reciproc files. J Endod 2017;43(3):462-466

13 Keskin C, Inan U, Demiral M, Keleş A. Cyclic fatigue resistance of reciproc blue, reciproc, and waveOne gold reciprocating instruments. J Endod 2017;43(8):1360-1363

14 Topçuoğlu HS, Topçuoğlu G. Cyclic fatigue resistance of reciproc blue and reciproc files in an s-shaped canal. J Endod 2017;43(10):1679-1682

15 Caballero-Flores H, Nabeshima CK, Binotto E, Machado MEL. Fracture incidence of instruments from a single-file reciprocating system by students in an endodontic graduate programme: a cross-sectional retrospective study. Int Endod J 2019;52(1):13-18
16 Muñoz E, Forner L, Llena C. Influence of operator's experience on root canal shaping ability with a rotary nickel-titanium single-file reciprocating motion system. J Endod 2014;40(4):547-550

17 Kwak SW, Cheung GS, Ha JH, Kim SK, Lee H, Kim HC. Preference of undergraduate students after first experience on nickel-titanium endodontic instruments. Restor Dent Endod 2016;41(3):176-181

18 Maniglia-Ferreira C, de Almeida Gomes F, Ximenes T, et al. Influence of reuse and cervical preflaring on the fracture strength of reciprocating instruments. Eur J Dent 2017;11(1):41-47 